\title{
HÁBITOS ALIMENTARIOS Y RIESGO \\ DE CANCER DE PIEL NO MELANOMA
}

\author{
DIET AND RISK OF NON \\ MELANOMA SKIN CANCER
}

María Susana Dagatti (1), Agustina Bertola Compagnucci (1), Stella Maris Pezzotto (1,2)

(1) Instituto de Inmunología. Facultad de Ciencias Médicas.

(2) Consejo de Investigaciones, Universidad Nacional de Rosario. Argentina.

\begin{abstract}
A case-control study was carried out in order to analyze the association between diet and risk of non melanoma skin cancer -basal cell carcinoma (BCC) and squamous cell carcinoma (SCC), with adjustments for demographic, anthropometric and phenotypic characteristics, sunburns history, skin cancer family history, sun-exposure history and skin sensitivity to sun exposure. A full-body skin examination was performed. Dietary data were obtained applying a standardized semi-quantitative questionnaire of consumption frequency. Cases (n=27; age: 65,5 $\pm 15,1$ years) and controls ( $n=37$; age: $63,9 \pm 12,3$ years) were attended at the same facilities. A decreased risk of $B C C$ and SCC tumors (Adjusted Odd Ratio=0.10; IC 95\%=0.02-0.63; $p=0.01$ ) was found for high intakes of green leafy vegetables (more than $40 \mathrm{gr} /$ day). However, results obtained for fruits, cruciferous, vitamin A and carotene-rich vegetables and other vegetables were not statistically significant.
\end{abstract}

Key words: Skin cancer; basal cell carcinoma; squamous cell carcinoma; risk factors; diet.

Este trabajo fue recibido el 7 de Julio de 2009 y aceptado para ser publicado el 20 de Noviembre de 2010.

\section{INTRODUCCIÓN}

El carcinoma basocelular (CBC) y el carcinoma Espinocelular (CEC) representan la mayor proporción de casos de cáncer de piel no melanoma. Ambos tienen su origen en el queratinocito, célula que se ubica en la epidermis cutánea (1). La incidencia de esta patología se encuentra en permanente ascenso en la mayoría de los países de occidente (2-4).

La asociación de esta patología con la exposición solar ha sido documentada en numerosas publicaciones, considerándose importante en su patogénesis tanto la exposición solar continua como la intermitente. Entre los factores de riesgo más importantes han sido estudiados el fototipo de piel, la historia de quemaduras solares, los antecedentes familiares de cáncer de piel y la presencia de lesiones previas, tales como queratosis actínicas y nevos atípicos (5-8).

Se ha documentado que la dieta jugaría un papel sustancial en el desarrollo de diversos tipos de cáncer. Datos epidemiológicos han revelado que el riesgo de desarrollar un tumor maligno presenta una gran variación entre poblaciones lo que estaría determinado en gran medida por factores ambientales y sería independiente de los factores genéticos poblacionales. Diversos estudios han demostrado la importancia de la nutrición en el desarrollo de cáncer, estimándose que hasta un $40 \%$ de los tumores podría ser prevenido a través de mantener una dieta adecuada y estilos de vida saludables (9). Se han obtenido evidencias de que los factores dietarios podrían prevenir el CEC y CBC, entre otros tumores cutáneos (10). Estudios in vitro y en animales sugieren que la ingesta de antioxidantes, como las vitaminas $\mathrm{C}$ y E y b-carotenos (11-13), podrían proteger contra el daño oxidativo a la piel, neutralizando ciertos radicales libres (14). En la actualidad la dieta se sigue investigando a fin de evaluar si ejercería un efecto protector en el desarrollo de este tipo de cáncer (15).

Este estudio se desarrolló en Rosario, uno de los mayores centros urbanos de la República Argentina, ciudad de aproximadamente 1.000 .000 de habitantes. La misma se halla situada a orillas del río Paraná y su clima es templado (Lat. 32`56’S). Debido a que los estudios 
epidemiológicos de dicha patología en esta ciudad son escasos, es necesario comenzar a investigar la incidencia de la misma para tener un real conocimiento del problema, que posiblemente sea subestimado.

El objetivo de este trabajo fue analizar la posible acción de la dieta como factor de riesgo de desarrollar cáncer cutáneo no melanoma de tipo CBC y CEC.

\section{SUJETOS Y MÉTODOS}

En la ciudad de Rosario, Argentina, se realizó un estudio epidemiológico analítico de casos y controles. Los casos fueron individuos de ambos sexos con diagnóstico clínico e histológico de CBC o CEC que concurrieron espontáneamente a la consulta dermatológica. Los controles fueron pacientes que asistieron a las mismas instituciones por patologías distintas a las mencionadas. Se excluyeron del estudio a los pacientes inmunodeprimidos, a aquellos que presentaron este tipo de tumor sobre úlceras o escaras previas, a quienes hubiesen realizado o estuvieran realizando tratamiento radiante, tratamientos con sustancias fotosensibilizantes, a los procedentes de áreas endémicas de hidroarsenicismo crónico y a quienes padecían el síndrome del Nevo Basocelular.

En la anamnesis realizada se consignaron datos demográficos y antropométricos, características fenotípicas, antecedentes familiares de cáncer de piel, exposición solar laboral, vacacional y en el tiempo libre, uso de lámparas UV o camas solares, quemaduras solares en la infancia, en la adolescencia y en la última década y uso de pantallas solares. Además se realizó un exhaustivo examen físico cutáneo.

Para clasificar a los pacientes según su tipo de piel de acuerdo al color y la facilidad para broncearse con el sol, se utilizó la clasificación del Dr. T. Fitzpatrick (16). Se trata de la observación del color de la piel en las partes sin exponer o expuestas mínimamente al sol. Según esta clasificación, se consideran seis tipos de piel: Tipo I - Presenta intensas quemaduras solares, casi no se pigmenta nunca y se descama de forma ostensible; Tipo II - Se quema fácil e intensamente, pigmenta ligeramente y descama de forma notoria.; Tipo III - Se quema moderadamente y se pigmenta correctamente.; Tipo IV - Se quema moderada o mínimamente y pigmenta con bastante facilidad y de forma inmediata al exponerse al sol.; Tipo V - Raramente se quema, pigmenta con facilidad e intensidad; Tipo VI - No se quema nunca y pigmenta intensamente.

Para determinar la exposición solar habitual se tomó en cuenta en forma conjunta el número de horas que los pacientes estuvieron expuestos al sol tanto en actividades cotidianas como laborales. Se consideró con exposición solar intermitente a los pacientes que realizaban actividades recreativas, vacacionales -al menos 10 días corridos en el año- y fines de semana. La categoría exposición solar mínima fue asignada a aquellos pacientes que no realizaban actividades laborables ni recreativas al aire libre, ni presentaban exposición solar vacacional.

La información sobre la dieta fue recolectada a través de un cuestionario de frecuencia de consumo (FFQ) semicuantitativo validado para su uso en Argentina (17). Para calcular los gramos de consumo diario de cada alimento, la cantidad de porciones se multiplicó por el gramaje de las mismas estandarizado para cada alimento en el FFQ, según lo reportado por cada encuestado y luego se convirtió a consumo diario. Posteriormente se calculó el contenido de cada nutriente a través de tablas de composición de alimentos (18).

Análisis estadístico: Para describir las variables cuantitativas se calcularon medias \pm errores estándar. La comparación de estos valores entre casos y controles se realizó aplicando pruebas t de student. La significación estadística de las diferencias en la ingesta promedio de cada nutriente analizado entre casos y controles se evaluó a través de un análisis de covarianza, ajustando por energía consumida. La significación de las diferencias entre proporciones se analizó utilizando pruebas de chicuadrado y prueba de la probabilidad exacta de Fisher, según correspondiera.

Se calculó la mediana del consumo de cada grupo de alimentos en el conjunto total de pacientes estudiados, y se la utilizó para dicotomizar cada una de estas variables en consumo bajo o alto. Se aplicó análisis de regresión logística múltiple para evaluar la asociación entre las variables de interés y el riesgo de esta patología, ajustando por el efecto de las demás variables intervinientes analizadas. Se estimaron los riesgos relativos a través del cálculo de los Odd-ratios (OR) como medidas de asociación entre el CBC y CEC y los factores de riesgo o de protección analizados. Se calcularon sus respectivos intervalos de confianza del 95\% (IC 95\%). Los datos fueron procesados utilizando el programa STATA 6.0.

Este estudio fue aprobado por la Comisión de Bioética de la Facultad de Ciencias Médicas de la Universidad Nacional de Rosario, y todos los participantes firmaron un formulario de consentimiento informado.

\section{RESULTADOS}

Se estudiaron 27 casos y 37 controles de ambos sexos, cuyos promedios de edad fueron $65,5 \pm 2,9$ y 63,9 $\pm 2,0$, respectivamente $(\mathrm{p}=0,643)$. Las características generales más relevantes de los pacientes estudiados se muestran en la tabla 1. El $44 \%$ de los casos y $51 \%$ de los controles eran de sexo masculino $(\mathrm{p}=0,58)$. El 
índice de masa corporal promedio (IMC) fue similar en los casos y en los controles ( $\mathrm{p}=0,323$ ). El 37,1\% de los casos y $64,8 \%$ de los controles tenían educación primaria (completa o incompleta) y el 62,9\% y 35,2\% tenían educación secundaria o superior, respectivamente $(\mathrm{p}=0,050)$. El 29,6\% de los casos y 43,2\% de los controles tenían exposición solar habitual, el resto de los pacientes tenía exposición solar intermitente $(63,0 \%$ y $24,3 \%)$ o mínima $(7,4 \%$ y $32,4 \%)$, respectivamente $(\mathrm{p}=0,004)$. El $51,9 \%$ de los casos y el 45,9\% de los controles tenían antecedentes de quemaduras solares $(\mathrm{p}=0,640)$. Ninguno de los pacientes, tanto casos como controles, había utilizado lámparas UV ni camas solares. No se encontraron pacientes con fototipo de piel V y VI, la distribución de los pacientes según esta variable fue significativamente diferente entre casos y controles $(\mathrm{p}=0,012)$.

$\mathrm{Al}$ analizar los resultados del examen físico cutáneo se encontró que las queratosis actínicas fueron significativamente más frecuentes $(\mathrm{p}=0,004)$ en los casos $(74,1 \%)$ que en los controles $(37,8)$. Además, el $66,7 \%$ de los casos y el $100 \%$ de los controles presentaban menos de 10 nevos ( $\mathrm{p}<0,001)$. El 18,5\% de los casos y $8,1 \%$ de los controles $(\mathrm{p}=0,194)$ tenían antecedentes familiares de cáncer de piel.

Las calorías totales consumidas por los casos fueron de $2055,4 \pm 124,4$ kcal. y por los controles de 1884,5 \pm $759,1 \mathrm{kcal}$. No se encontró diferencia estadísticamente significativa en la energía total consumida promedio entre los casos y los controles $(\mathrm{p}=0,348)$. El consumo promedio de los diferentes de grupos de alimentos se

\section{TABLA 1}

Características generales de los casos y controles.

Variable

Sexo

Masculino

Femenino

Edad

Promedio \pm desvío estándar

Rango

IMC $\left(\mathrm{Kg} / \mathrm{m}^{2}\right)$

Promedio \pm desvío estándar

Rango

Exposición solar

Habitual

Intermitente

Mínima

\section{Quemaduras solares previas}

Fototipo de piel

Tipo I

Tipo II

Tipo III

Tipo IV

Queratosis actínicas

Menos de 10 Nevos

Antecedentes familiares de cáncer de piel
Casos
n $(\%)$
Controles
n $(\%)$

p

$0,585^{1}$

$18(48,6)$

$\begin{array}{cc}65,5 \pm 15,1 & 63,9 \pm 12,3 \\ 32-90 & 36-87 \\ & \\ 28,0 \pm 5,5 & 26,8 \pm 4,0 \\ 21-47 & 20-40\end{array}$

$16(43,2)$

$9(24,3)$

$12(32,4)$

$17(45,9)$

$0,640^{1}$

24,3

$0,012^{3}$

37,8

27,0

10,8

37,8

$0,004^{1}$

100,0

$<0,001^{1}$

8,1

${ }^{1}$ Prueba Chi-cuadrado; ${ }^{2}$ Pruebas t de Student; ${ }^{3}$ Prueba de tendencia lineal. 
muestra en la tabla 2, en la que se observa que los casos presentaron una tendencia a consumir más lácteos, grasas y crucíferas (repollo, coliflor, brócoli, repollitos de Bruselas) y menos vegetales de hojas verdes (espinaca, lechuga, apio y acelga) que los controles.

Tal como se expresara en la sección materiales y métodos, se realizó un análisis basado en modelos de regresión logística múltiple para evaluar el efecto de cada variable en forma individual sobre el riesgo de cáncer de piel no melanoma, ajustado por los posibles efectos confundidores de las demás variables involucradas. Los OR ajustados mediante esta técnica, comparando consumo alto versus bajo de los grupos de alimentos estudiados se muestran en la tabla III. Los OR correspondientes a consumo alto de frutas, crucíferas, vegetales ricos en vitamina A y carotenos (zapallo, calabaza, batata, tomate y zanahoria) y otros vegetales (papa, remolacha, cebolla, ajo, pimiento, ajíes enlatados y berenjenas) no resultaron estadísticamente significativos. En cambio, la ingesta alta (más de 40 gramos diarios) de vegetales de hojas verdes estaría actuando como un factor protector significativo.

Además, se encontraron disminuciones en el riesgo de desarrollar esta patología con la exposición solar habitual versus la intermitente $(\mathrm{OR}=0,16 ; \mathrm{p}=0,049$; IC $95 \%=0,03-0,99)$ y con la exposición solar mínima versus la intermitente $(\mathrm{OR}=0,26 ; \mathrm{p}=0,043 ; \mathrm{IC} 95 \%=0,07-0,96)$ $\mathrm{y}$ un incremento del riesgo con la presencia de queratosis actínicas $(\mathrm{OR}=2,33 ; \mathrm{p}=0,01 ; \mathrm{IC} 95 \%=1,24-4,39)$.

\section{DISCUSIÓN}

El cáncer de piel es más frecuente que cualquier otro tipo de cáncer y se ha documentado que la exposición solar contribuye a desarrollar las tres variedades de mayor incidencia, como lo son el CBC, CEC y el melanoma cutáneo (6).

Numerosos estudios examinaron la relación entre el riesgo de desarrollar CBC según los factores constitucionales de las personas y su exposición solar, hallando asociación positiva entre el número de quemaduras solares y este cáncer. La piel, cabello y ojos claros y la edad avanzada serían importantes factores de riesgo (19-21). Otros estudios han sido realizados a fin de analizar si la exposición solar de tipo laboral diaria o la ocasional incidirían de distinta manera como factores de riesgo tanto para el CBC como para el CEC (22-26).

Davies y colaboradores plantean que se ha atribuido el aumento de la incidencia de CBC y CEC al incremento de la exposición solar. Sin embargo, dicho factor no ha sido aun suficientemente cuantificado. Esta patología se ha manifestado principalmente en cabeza y cuello, zonas siempre mas expuestas a la radiación solar. Estos autores

\section{TABLA 2}

Consumo promedio (media \pm error estándar) de los diferentes de grupos de alimentos.

\begin{tabular}{|c|c|c|c|c|c|}
\hline Alimentos (gramos/día) & $\operatorname{Casos}^{\mathrm{a}}$ & Controles $^{\mathrm{a}}$ & Casos $^{b}$ & Controles $^{b}$ & $\mathbf{p}^{\mathbf{c}}$ \\
\hline \multicolumn{6}{|l|}{ Vegetales ricos en vitamina $\mathrm{A}$} \\
\hline y carotenos ${ }^{1}$ & $135,7 \pm 16,4$ & $126,6 \pm 14,5$ & $134,3 \pm 16,9$ & $127,7 \pm 14,4$ & 0,768 \\
\hline Vegetales de hojas verdes ${ }^{2}$ & $15,4 \pm 4,2$ & $62,8 \pm 13,0$ & $16,2 \pm 12,0$ & $62,2 \pm 10,0$ & 0,005 \\
\hline Crucíferas $^{3}$ & $32,8 \pm 5,4$ & $15,4 \pm 6,1$ & $32,6 \pm 6,6$ & $15,5 \pm 5,6$ & 0.053 \\
\hline Otros vegetales ${ }^{4}$ & $78,9 \pm 13,4$ & $92,7 \pm 10,7$ & $78,2 \pm 13,1$ & $93,2 \pm 11,1$ & 0.389 \\
\hline Frutas ricas en vitaminas $\mathrm{A}$ y $\mathrm{C}^{5}$ & $104,1 \pm 19,9$ & $105,3 \pm 22,0$ & $102,8 \pm 23,7$ & $106,3 \pm 20,3$ & 0,911 \\
\hline Otras frutas & $178,6 \pm 20,1$ & $145,8 \pm 16,8$ & $180,3 \pm 20,0$ & $144,5 \pm 17,1$ & 0,181 \\
\hline Carnes y fiambres & $195,6 \pm 19,0$ & $204,8 \pm 23,4$ & $185,9 \pm 20,5$ & $211,8 \pm 17,5$ & 0,344 \\
\hline Lácteos & $398,8 \pm 42,0$ & $281,4 \pm 32,3$ & $395,2 \pm 39,9$ & $284,0 \pm 34,0$ & 0,039 \\
\hline Huevos & $7,5 \pm 0,9$ & $8,1 \pm 2,3$ & $7,2 \pm 2,1$ & $8,4 \pm 1,8$ & 0,680 \\
\hline Otras grasas & $8,4 \pm 3,0$ & $3,1 \pm 1,0$ & $8,3 \pm 2,2$ & $3,2 \pm 1,9$ & 0,077 \\
\hline
\end{tabular}


sugieren que existe evidencia de que el incremento de la ingesta de una dieta rica en grasas ha intensificado la sensibilidad de la piel a potenciales cancerígenos como la luz solar, particularmente en el CEC (27). Se debe tener presente que la dieta habitual en nuestro país presenta dichas características.

Desde hace algunos años se viene estudiando un grupo específico de vitaminas con ciertas propiedades y funciones en común, los antioxidantes. Estos se caracterizan por tener la función de impedir o retrasar la oxidación de diversas sustancias, principalmente de los ácidos grasos cuyas reacciones se producen tanto en los alimentos como en el organismo humano, pudiendo provocar en este último alteraciones fisiológicas importantes (28).

El mecanismo primario por el cual las vitaminas A y E tendrían un efecto protector en la carcinogénesis puede deberse a que actúan como antioxidantes y protectores celulares contra el daño oxidativo, no descartándose la existencia de otros posibles mecanismos involucrados (29). Se sabe además que la vitamina A tendría ciertos efectos sobre el sistema inmune (30).

La importancia de los factores dietéticos sobre el desarrollo de CBC y CEC ha sido recientemente estudiada en una revisión sistemática de 26 trabajos publicados (31). Dicho análisis indicó que existiría una asociación positiva entre estas patologías y la ingesta de una dieta rica en grasas, una asociación inconsistente con el retinol y una asociación leve con los b-carotenos. La evidencia para la asociación con la vitamina E, vitamina $\mathrm{C}$, y el selenio fue débil. Los autores plantean que para clarificar estos puntos sería necesaria la realización de nuevos estudios.

Se conoce que las verduras de hojas verdes contienen una variedad de vitaminas, minerales y otras sustancias bioactivas que pueden proteger contra cánceres, tales como la luteína, las vitaminas C, E y ácido fólico, flavonoides y fibras. Las verduras de hojas verdes ejercerían un efecto protector contra el desarrollo de CEC; que podría deberse a que la combinación de nutrientes encontrada en estos alimentos proporciona un efecto anti-cancerígeno, o al hecho de que la ingesta de este tipo de verduras estaría indicando un modelo de dieta saludable. En un estudio realizado en Nambour se encontró una correlación significativa entre el consumo de verduras de hojas verdes y el consumo de otras frutas, otras verduras y pollo sin piel. Los autores sugieren que el consumo de verduras de hojas verdes, que son una fuente rica de ácido fólico, jugaría un papel importante en la síntesis, reparación y metilación del ADN (32).

Como mencionamos previamente, la exposición solar es el factor de riesgo más importante para desarrollar

\section{TABLA 3}

\section{OR e intervalos de confianza del $95 \%$ para CBC y CCE según consumo de los distintos grupos de alimentos.}

\section{Grupos de alimentos}

Vegetales ricos en vitamina A y carotenos 1 ( $\geq 100$ g/día)

Vegetales de hojas verdes 2 ( $\geq 40 \mathrm{~g} /$ día)

Crucíferas3 ( $\geq 20 \mathrm{~g} /$ día)

Otros vegetales4 ( $\geq 80 \mathrm{~g} /$ día)

Frutas ricas en vitaminas A y C5 ( $\geq 70 \mathrm{~g} / \mathrm{día})$

Otras frutas ( $\geq 130 \mathrm{~g} /$ día)

Carnes y fiambres ( $\geq 160 \mathrm{~g} /$ día $)$

Lácteos ( $\geq 325 \mathrm{~g} /$ día)

Grasas ( $\geq 10 \mathrm{~g} / \mathrm{día})$

Huevos ( $\geq 10$ g/día)

OR $^{*} \quad$ IC 95\%

p

1,33

0,10

1,32

0,96

1,02

1,17

0,92

2,71

1,35

1,43
0,47-3,76

0,02-0,63

0,98-2,04

$0,35-2,64$

0,37-2,81

0,43-3,18

0,31-2,75

0,97-7,61

0,49-3,75

$0,45-4,53$
0,59

0,01

0,25

0,94

0,96

0,76

0,88

0,06

0,57

0,54

${ }^{1}$ zapallo, calabaza, batata, tomate y zanahoria; ${ }^{2}$ espinaca, lechuga, apio y acelga; ${ }^{3}$ repollo, coliflor, brócoli, repollitos de Bruselas; ${ }^{4}$ papa, remolacha, cebolla, ajo, pimiento, ajíes enlatados y berenjenas; ${ }^{5}$ cítricos, kiwi, ciruelas y cerezas.

"Estimados a partir de un modelo logístico incluyendo los grupos de alimentos, la energía total consumida, la exposición solar, el fototipo de piel (I-II/III-IV) y la presencia de queratosis actínicas. 
CEC o CBC pero, a pesar de ello, un alto consumo de vegetales de hojas verdes modificaría el efecto negativo de este factor.

Una limitación del presente estudio es el reducido número de pacientes incorporados hasta este momento. Las ventajas del mismo están dadas por una recolección minuciosa de información sobre numerosas variables, considerando no sólo las características fenotípicas de las personas entrevistadas, sus hábitos de exposición solar y estilo de vida sino también las características de la dieta. Se debe tener presente que la anamnesis alimentaria requiere un cuidadoso entrenamiento del entrevistador y la realización de la misma demanda un tiempo importante. El FFQ es el método más apropiado para la obtención de datos sobre alimentación en estudios epidemiológicos, debido a que permite categorizar a los individuos en función del nivel de consumo de alimentos, por ende puede discriminar aquellas personas que consumen mucho un alimento o grupo de ellos en relación a las que lo consumen poco o no lo hacen nunca. Además, este método posee la característica de ser práctico, económico y proveer una adecuada información sobre antiguas ingestas alimentarias (33).

Los resultados preliminares de este estudio permiten identificar grupos de riesgo en los cuales la implementación de medidas preventivas simples tendría un rol fundamental. Es necesario insistir en mejorar la educación acerca de los peligros del sol, así como la importancia de una dieta saludable.

\section{RESUMEN}

Mediante un diseño de casos y controles se evaluó si la dieta habitual modifica el riesgo de desarrollar cáncer de piel no melanoma: carcinomas basocelulares y carcinomas espinocelulares. En la consulta se consignaron datos demográficos, características fenotípicas y antropométricas, antecedentes de quemadura solar, antecedentes familiares de cáncer de piel y hábitos de exposición solar, y se realizó un exhaustivo examen físico cutáneo. La dieta fue evaluada por cuestionarios semi-cuantitativos de frecuencia de consumo. Se estudiaron 27 casos (edad: $65,5 \pm 15,1$ años) y 37 controles $(63,9 \pm 12,3)$ que asistieron a las mismas instituciones por otras patologías. La ingesta alta de vegetales de hojas verdes (más de $40 \mathrm{~g} / \mathrm{d}$ ) actuaría como factor protector (Odd Ratio ajustado=0,10; IC 95\%=0,02-0,63; p=0,01), modificando el efecto negativo de la exposición solar. En cambio, los resultados obtenidos para frutas, crucíferas, vegetales ricos en vitamina A y carotenos y otros vegetales no resultaron estadísticamente significativos.

Palabras clave: Cáncer cutáneo; carcinoma basocelular; carcinoma espinocelular; factores de riesgo; dieta.
Dirigir la correspondencia a:

Dra.

Stella M. Pezzotto

Instituto de Inmunología,

Facultad de Ciencias Médicas

Santa Fe 3100.

2000 Rosario

Argentina

Teléfono: + 54-341-155669150

Fax: + 54-341-4804569

E-mail: spezzot@unr.edu.ar

\section{BIBLIOGRAFÍA}

1. Jemal A, Murray T, Samuels A, Ghafoor A, Ward E, Thun MJ. Cancer statistics 2003. CA Cancer J Clin. 2003; 53(1):5-26.

2. Greenlee RT, Hill-Harmon MB, Murray T, Thun M. Cancer statistics 2001. CA Cancer J Clin. 2001; 51:15-36.

3. Michal Freedman D, Sigurdson A, Morin Doody M, Mabuchi K, Linet MS. Risk of Basal Cell Carcinoma in Relation to Alcohol Intake and Smoking. Cancer Epidemiology, Biomarkers Prevention. 2003; 12:1540-1543.

4. Cantwell MM, Murray LJ, Catney D, Donnelly D, Autier P, Boniol M, Fox C, Middleton RJ, Dolan OM, Gavin AT. Second primary cancers in patients with skin cancer: a population-based study in Northern Ireland Br J Cancer. 2009 13; 100(1): 174-7.

5. Adami H.O, Hunter D, Trichopoulos D. Skin Cancer in Textbook of cancer epidemiology. Oxford University Press 2002; Chap 13:281-301.

6. Turgay AS, Sari D, Can M, Genc RE. Determination of sunburn and skin cancer risk of outpatients in a dermatology polyclinic. Asian Pac J Cancer Prev. 2005;6(2):143-6.

7. Scotto J, Fears TR, Kraemer KH, Fraumeni JF Jr. Nonmelanoma skin cancer, in: Schottenfeld, D and Fraumeni, JF Jr. (eds.), Cancer Epidemiology and Prevention, 2nd Ed. New York: Oxford University Press, 1996

8. Leiter U, Garbe O Epidemiology of melanomaand nonmelanomaskin cancer--the role of sunlight. Adv Exp Med Biol. 2008;624:89-103.

9. Pierart C, Rozowsky J. Papel de la nutrición en la prevención del cáncer gastrointestinal. Rev Chil Nutr 2006; 33 (1): 8-13.

10. Ibiebele TI, van der Pols JC, Hughes MC, Marks GC, Williams GM, and Green A C. Dietary pattern in association with squamous cell carcinoma of the skin: a prospective study. Am J Clin Nutr. 2007; 85:1401-8. 
11. Rackett SC, Rothe MJ, Grant-Kels JM. Diet and dermatology. The role of dietary manipulation in the prevention and treatment of cutaneous disorders. J Am Acad Dermatol 1993:29:447-61

12.

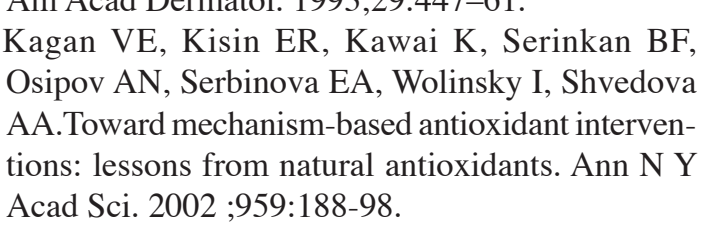

13. Sies H, Stahl W. Nutritional protection against skin damage from sunlight. Annu Rev Nutr. 2004;24:173-200.

14. Steenvoorden DP, Van Henegouwen GM. The use of endogenous antioxidants to improve photoprotection. J Photochem Photobiol B. 1997;41:1-10.

15. Fung TT, Hunter HJ, Spiegelman D, Colditz GA, Speizer FE, Willett WC. Vitamins and carotenoids intake and the risk of basal cell carcinoma of the skin in women. Cancer Causes Control. 2002;13: 221-30.

16. Fitzpatrick T. Dermatología en Medicina General $7^{\text {a }}$ Ed. Médica Panamericana, 2009.

17. Navarro A, Osella AR, Guerra V, Muñoz SE, Lantieri MJ, Eynard AR. Reproducibility and validity of a food-frequency questionnaire in assessing dietary intakes and food habits in epidemiological cancer studies in Argentina. J Exp Clin Cancer Res. 2001;20(3):365-70.

18. Composition of Foods.: Raw, Processed, Prepared U.S. Department of Agriculture, Agricultural Research Service, USDA Nutrient Data Laboratory. USDA Nutrient Database for Standard Reference, Release p. 14, 2001.

19. Saladi RN, Persaud AN. The causes of skin cancer: a comprehensive review. Drugs Today (Barc). 2005; 41:37.

20. Van Dam RM, Huang Z, Rimm EB, Weinstock MA, Spiegelman D, Colditz GA, Willett WC, Giovannucci E. Risk factors for basal cell carcinoma of the skin in men: results from the health professionals follow-up study. Am J Epidemiol. 1999; 150(5):459-68.

21. Terkonda SP, Perdikis G. Non-melanotic skin tumors of the upper extremity. Hand Clin. 2004; 20(3):293-301.
22. Pipitone M, Robinson JK, Camara C, Chittineni B, Fisher SG. Skin cancer awareness in suburban employees: a Hispanic perspective. J Am Acad Dermatol 2002:47(1):118-23

23. Badespiel-Tröger M Meyer M, Pfahlberg A, Lausen B. Uter W. Gefeller O Outdoor work and skin canfer incidence. a registry-hased study in Bavaria. Int Arch Occup Environ Health. 2009;82(3):357-63.

24. Almahroos M, Kurban AK. Ultraviolet carcinogenesis in nonmelanoma skin cancer part II: review and update on epidemiologic correlations. Skinmed. 2004;3(3):132-9.

25. Heenan MG, Randell PJ. Case-control study of sun exposure and squamous cell carcinoma of the skin. Int J Cancer. 1998; 77(3):347-53.

26. Kricker A, Armstrong BK, English DR, Heenan, PJ. Does intermittent sun exposure cause basal cell carcinoma? A case-control study in Western Australia. Int J Cancer. 1995; 60(4):489-94.

27. Davies TW, Treasure FP, Welch AA, Day NE. Diet and basal cell skin cancer: results from the EPICNorfolk cohort. Br J Dermatol. 2002; 146(6):101722.

28. Zamora JD. Antioxidantes: micronutrientes en lucha por la salud. Rev Chil Nutr 2007; 34(1): 17-26.

29. McNaughton SA, Marks GC, Gaffney P, Williams G, Green AC.: Antioxidants and basal cell carcinoma of the skin: a nested case-control study. Cancer Causes Control 2005:16(5):609-18.

30. La Vecchia C, Altieri A, Tavani A. Vegetables, fruit, antioxidants and cancer: a review of Italian studies. Eur J Nutr. 2001;40(6):261-7.

31. McNaughton SA, Marks GC, Green AC. Role of dietary factors in the development of Basal Cell Cancer and Squamous Cell Cancer of the Skin. Cancer Epidemiol Biomarkers Prev. 2005;14(7):1596-607.

32. Hughes MC, van der Pols JC, Marks GC, Green. AC. Food intake and risk of squamous cell carcinoma of the skin in a community: The Nambour skin cancer cohort study. Int J Cancer 2006;119:1953-60.

33. Willett WC, Hu FB. Not the Time to Abandon the Food Frequency Questionnaire. Cancer Epidemiol Biomarkers Prev. 2006; 15: 1757-8. 